\title{
Regulation of Neurotransmitter Release by Synapsin III
}

\author{
Jian Feng, ${ }^{1}$ Ping Chi, ${ }^{1,3}$ Thomas A. Blanpied, ${ }^{4}$ Yimei Xu, ${ }^{4}$ Ana Maria Magarinos, ${ }^{2}$ Adriana Ferreira, ${ }^{5}$ \\ Reisuke H. Takahashi, ${ }^{1}$ Hung-Teh Kao, ${ }^{1,6}$ Bruce S. McEwen, ${ }^{2}$ Timothy A. Ryan, ${ }^{3}$ George J. Augustine, ${ }^{4}$ and \\ Paul Greengard 1
}

1 Laboratory of Molecular and Cellular Neuroscience and 2Laboratory of Endocrinology, The Rockefeller University, New York, New York 10021, 32Department of Biochemistry, The Weill Medical College of Cornell University, New York, New York 10021, 4Department of Neurobiology, Duke University Medical Center, Durham, North Carolina 27710, 5/nstitute for Neuroscience, Northwestern University, Chicago, Illinois 60611, and ${ }^{6}$ Department of Psychiatry, New York University Medical College, New York, New York 10016

Synapsin III is the most recently identified member of the synapsin family, a group of synaptic vesicle proteins that play essential roles in neurotransmitter release and neurite outgrowth. Here, through the generation and analysis of synapsin III knock-out mice, we demonstrate that synapsin III regulates neurotransmitter release in a manner that is distinct from that of synapsin I or synapsin II. In mice lacking synapsin III, the size of the recycling pool of synaptic vesicles was increased, and synaptic depression was reduced. The number of vesicles that fuse per action potential was similar between synapsin III knock-out and wild-type mice, and there was no change in the quantal content of EPSCs; however, IPSCs were greatly re- duced in synapsin III-deficient neurons. The density and distribution of synaptic vesicles in presynaptic terminals did not appear to be different in synapsin III knock-out mice in comparison to wild-type littermates. In addition to the changes in neurotransmitter release, we observed a specific delay in axon outgrowth in cultured hippocampal neurons from synapsin III knock-out mice. Our data indicate that synapsin III plays unique roles both in early axon outgrowth and in the regulation of synaptic vesicle trafficking.

Key words: synapsin III; knock-out; synaptic transmission; synaptic vesicle trafficking; neurotransmitter release; neurite outgrowth
Synapsins are a group of neuron-specific phosphoproteins that are associated with synaptic vesicles (Greengard et al., 1993). Three mammalian synapsin genes have been identified and named synapsins I, II, and III. Transcripts of each of these genes are differentially spliced to give rise to various isoforms (Sudhof et al., 1989; Hosaka and Sudhof, 1998; Kao et al., 1998; Porton et al., 1999). Previous studies have demonstrated that synapsins play critical roles in anchoring synaptic vesicles to the cytoskeletal network of presynaptic terminals (Hirokawa et al., 1989; Bahler et al., 1990; Llinas et al., 1991; Benfenati et al., 1992; Pieribone et al., 1995). Phosphorylation of synapsins by various kinases changes the affinity between synapsins and actin filaments (Bahler and Greengard, 1987) and may allow mobilization of vesicles at synapses (Huttner et al., 1981; Valtorta et al., 1992; Jovanovic et al., 1996; Matsubara et al., 1996; Chi et al., 2001). Synaptic depression is particularly disrupted after either acute disruption of synapsin function (Pieribone et al., 1995; Hilfiker et al., 1998) or ablation of synapsin genes (Li et al., 1995; Takei et al., 1995), consistent with the notion that synapsins are involved

Received Oct. 4, 2001; revised March 13, 2002; accepted March 18, 2002.

This work was supported by National Institutes of Health Grants MH39327 and AG15072 (P.G.), NS17771 (G.J.A.), and NS24692 and GM61925 (T.A.R.), a Theodore and Vada Stanley Foundation Research Award (P.G., J.F.), a McKnight Foundation Award (G.J.A.), and a National Alliance for Research on Schizophrenia and Depression Young Investigator Award (A.F.). We thank Cassandra M. Kirk for excellent technical assistance, and The Rockefeller University Transgenic Animal Service for blastocyst injections.

Correspondence should be addressed to Dr. Jian Feng, Department of Physiology and Biophysics, State University of New York at Buffalo, 124 Sherman Hall, Buffalo, NY 14214. E-mail: jianfeng@buffalo.edu.

Copyright (C) 2002 Society for Neuroscience $\quad 0270-6474 / 02 / 224372-09 \$ 15.00 / 0$ in regulating the supply of vesicles available for release during periods of high activity.

Given the existence of three different synapsin genes, it is important to identify the unique roles that each plays. Mice lacking synapsin I, synapsin II, or both gene products have greatly decreased numbers of synaptic vesicles and exhibit significant changes in neurotransmitter release and synaptic depression $(\mathrm{Li}$ et al., 1995; Rosahl et al., 1995; Takei et al., 1995; Ryan et al., 1996). These changes indicate important presynaptic functions for synapsins I and II. The function of synapsin III, the latest member of the synapsin family to be discovered, is less clear. Despite its similar domain structure and sequence homology with synapsin I and synapsin II, synapsin III has several unique properties suggesting that the function of this protein is not redundant. The expression level of synapsin III, although generally much lower in adults than that of synapsin I or II (Kao et al., 1998), is developmentally regulated in a manner different from the other synapsins, declining after the first week postnatally (Ferreira et al., 2000). There is evidence that synapsin III regulates the rate of axonal growth and size of the growth cones of developing neurons (Ferreira et al., 2000). The subcellular localization of synapsin III is also unique: although synapsins I and II are localized almost exclusively to presynaptic terminals, synapsin III is found in cell bodies and growth cones as well as at presynaptic sites (Ferreira et al., 2000). Finally, $\mathrm{Ca}^{2+}$ inhibits the binding of ATP to synapsin III, whereas it activates the binding of ATP to synapsin I, and does not affect the binding of ATP to synapsin II (Hosaka and Sudhof, 1998).

The unique properties of synapsin III suggest that it plays a role different from those of synapsin I and synapsin II. Here, through 
the generation of synapsin III knock-out mice, we have analyzed in vivo functions of synapsin III in neurotransmitter release and neurite outgrowth. We find that synapsin III is involved in regulating the size of the recycling vesicle pool, the strength of synaptic depression, and very early axon outgrowth. These functions of synapsin III differ from those of synapsins I and II, indicating unique roles for each member of the synapsin gene family in neuronal function.

\section{MATERIALS AND METHODS}

Generation of synapsin III knock-out mice. Standard gene-targeting procedures were used to generate synapsin III knock-out mice essentially as described before (Feng et al., 2000). Briefly, genomic DNA containing exon 1 of the mouse synapsin III gene was cloned by screening a BAC genomic DNA library from Genome Systems, Inc. (St. Louis, MO). A targeting vector was constructed by using a $6.0 \mathrm{~kb} H i n \mathrm{dIII}-X$ XoI fragment as left arm and a $4.7 \mathrm{~kb}$ Sst $\mathrm{I}-P s t \mathrm{I}$ fragment as right arm for homologous recombination. The $2.0 \mathrm{~kb}$ XhoI-Sst I fragment containing exon 1 was replaced by a neomycin resistance gene in reverse orientation. After homologous recombination in RW4 embryonic stem (ES) cells (Genome Systems, Inc.), the targeted allele contained a $6.8 \mathrm{~kb} E c o$ RI fragment, whereas the wild-type allele had an $8.1 \mathrm{~kb}$ EcoRI fragment. Ten positive ES clones were obtained of the 480 clones picked (targeting ratio $\sim 2 \%$ ). Four of the positive clones were injected into C57BL/6 blastocysts and produced germ-line transmission of the targeted allele. Southern blots were performed on Eco RI digests of tail DNA with [ $\left.{ }^{32} \mathrm{P}\right]-\mathrm{dCTP}$-labeled external probe. Heterozygotes were intercrossed to generate wild-type and synapsin III knock-out mice used in the studies. Animal use and care were in strict accordance with protocols approved by the Institutional Animal Care and Use Committees at the investigators' universities.

Western blot. Mouse whole brain was homogenized in 1\% SDS solution and boiled for $5 \mathrm{~min}$. Protein concentration was measured by the DC Protein Assay kit (Bio-Rad, Hercules, CA). An equal amount of total proteins from each genotype was separated on SDS-polyacrylamide gel and transferred to nitrocellulose membranes (Invitrogen, Gaithersburg, MD) for Western blot analyses. The following antibodies were used: RU486 (against amino acids 544-558 of rat synapsin III sequence) and RU482 (against amino acids 446-464 of rat synapsin III sequence), G304 (pansynapsin antibody) (Kao et al., 1998), and RU87 (anti-synaptotagmin), anti-syntaxin and anti-synaptophysin (Sigma, St. Louis, MO).

Electron microscopy. Mice ( $n=5$ for each genotype) were anesthetized with Metofane (Pitman-Moore, Mundelein, IL) and transcardially perfused with saline followed by fixative containing $2 \%$ glutaraldehyde and $2 \%$ paraformaldehyde in $0.1 \mathrm{~m}$ phosphate buffer $(\mathrm{PB}), \mathrm{pH}$ 7.4. After the brain was incubated in the fixative solution overnight at $4^{\circ} \mathrm{C}$, coronal sections $(100 \mu \mathrm{m}$ thick) through the dorsal hippocampus were cut and postfixed in $2 \%$ osmium tetroxide in PB and partially dehydrated. Sections were stained with $2 \%$ uranyl acetate in $70 \%$ ethanol, further dehydrated, incubated with propylene oxide, and flat embedded in Durcupan (Fluka, Switzerland). The mossy fiber termination zone (stratum lucidum) was trimmed, and ultrathin silver sections were cut, mounted, and counterstained with Reynolds' lead citrate. The final preparations were examined and photographed with a Jeol $100 \mathrm{CX}$ electron microscope. Photographs (10 per subject) were randomly taken from the stratum lucidum area at a primary magnification of $5400 \times$. Prints $(13,500 \times)$ were used to trace mossy fiber terminals (MFTs) as well as mitochondria and spine profiles. An average of 60 MFTs per block per experimental animal were analyzed. The total MFT area as well as the areas occupied by mitochondria and spine profiles were measured with the aid of a Zeiss Interactive Digitizing System. Synaptic vesicle density was estimated using the intersection method, positioning an unbiased counting frame with squares of known area $\left(0.2 \mathrm{~cm}^{2}\right)$ at the coordinates of a quadratic lattice superimposed on the photomicrographs containing the MFTs. The number of vesicles per MFT unit area was averaged from counts obtained from five frames, and counts were adjusted to consider section thickness and lost caps by using a variation of Floderus's equation (Magarinos et al., 1997). Each variable was averaged across MFTs to obtain a single mean value per animal, and two-tailed unpaired Student's $t$ test was applied to assess statistical significance.

Neuronal cultures. For studies using FM1-43, hippocampal CA1-CA3 regions were dissected from wild-type or synapsin III knock-out mice at postnatal day 1 . Neurons were dissociated and plated onto polyornithinecoated coverslips inside a 5-mm-diameter cloning cylinder (100 $\mu \mathrm{l} \mathrm{vol}$;
Bellco Glass, Vineland, NJ). Cells were maintained in culture media consisting of MEM (Invitrogen), $0.6 \%$ glucose, $0.1 \mathrm{gm} / 1$ bovine transferrin (Calbiochem, La Jolla, CA), $0.25 \mathrm{gm} / 1$ insulin, $0.3 \mathrm{gm} / 1$ glutamine, 5-10\% fetal calf serum (HyClone, Logan, UT), 2\% B-27 (Invitrogen), and $8 \mathrm{~mm}$ cytosine $\beta$-D-arabinofuranoside (Sigma). Cultures were maintained at $37^{\circ} \mathrm{C}$ in a $95 \%$ air $/ 5 \% \mathrm{CO}_{2}$ humidified incubator for $14-15 \mathrm{~d}$ before use.

For axon development studies, neuronal cultures were prepared from the hippocampi of embryonic day 16 mice as described previously (Goslin and Banker, 1991; Ferreira et al., 2000). Briefly, embryos were removed, and their hippocampi were dissected and freed of meninges. The cells were dissociated by trypsinization $\left(0.25 \%\right.$ for $15 \mathrm{~min}$ at $\left.37^{\circ} \mathrm{C}\right)$ followed by trituration with a fire-polished Pasteur pipette and plated onto poly-L-lysine-coated coverslips in MEM with $10 \%$ horse serum. After $4 \mathrm{hr}$, the coverslips were transferred to dishes containing an astroglial monolayer and maintained in MEM containing N2 supplements plus ovalbumin $(0.1 \%)$ and sodium pyruvate $(0.1 \mathrm{~mm})$.

For patch-clamp recordings, autapses (Bekkers and Stevens, 1991; Tong and Jahr, 1994) were prepared by coating coverslips with $0.15 \%$ agarose (Sigma), drying these for $1 \mathrm{hr}$, and then spraying them with a fine mist of poly-D-lysine $(0.5 \mathrm{mg} / \mathrm{ml})$ and collagen $(0.5 \mathrm{mg} / \mathrm{ml})$. Isolated hippocampi of 1- to 2-d-old mice were treated with papain, and the dissociated cells were plated at $2000-5000 \mathrm{~cm}^{-2}$ in minimal essential media (Invitrogen) supplemented with 5\% FBS (Invitrogen), B-27 (Invitrogen), and $\mathrm{Mito}^{+}$serum extender (Collaborative Biomedical) at their suggested concentrations, $3.6 \mathrm{gm} / 1$ glucose, $1 \%$ Pen/Strep (Invitrogen), and $500 \mu \mathrm{M}$ MEM sodium pyruvate (Invitrogen). This medium was exchanged $24 \mathrm{hr}$ after plating and again after $6 \mathrm{~d}$ in culture, and cells were used for recordings after 7-9 $d$ in culture.

Electrophysiology procedures. For patch-clamp recording, cells were bathed in solution containing (in $\mathrm{mM}$ ): $150 \mathrm{NaCl}, 3 \mathrm{KCl}, 2 \mathrm{CaCl}_{2}, 2$ $\mathrm{MgCl}_{2}, 20$ glucose, $10 \mathrm{HEPES}$, and $50 \mu \mathrm{M}$ DL-APV. To avoid bias toward particular types of neurons, a recording was attempted from each healthy neuron that was morphologically isolated from all other neurons (to ensure recording responses from autapses rather than synapses between neurons). Patch pipettes (3-4 M $\Omega$ ) were filled with an intracellular solution containing (in $\mathrm{mM}$ ): $71 \mathrm{~K}$-gluconate, $50 \mathrm{~L}$-glutamate, $6 \mathrm{MgCl}_{2}, 5$ $\mathrm{Na}_{2}$ ATP, $0.3 \mathrm{Na}_{2}$ GTP, 2 EGTA, and 20 HEPES, with the $\mathrm{pH}$ adjusted to 7.3 with KOH. An EPC-9 patch-clamp amplifier was used to record synaptic currents, which were filtered at $1.5 \mathrm{kHz}$, digitized at $10 \mathrm{kHz}$, and analyzed off-line with custom software. Neurons were held at a potential of $-70 \mathrm{mV}$; series resistance ranged from 5 to $20 \mathrm{M} \Omega$, and $80 \%$ of this resistance was compensated electronically.

Synaptic transmission was evoked at $15 \mathrm{sec}$ intervals by delivering voltage steps to $+10 \mathrm{mV}(0.1 \mathrm{msec}$ duration $)$ that initiated unclamped, axonal action potentials. The resulting PSCs were measured between 0.5 and $2.5 \mathrm{~min}$ after the whole-cell configuration was established. PSCs could be classified as excitatory or inhibitory on the basis of their time courses of decay and their reversal potentials. One group of PSCs had a mean decay time constant of $4.3 \pm 0.2 \mathrm{msec}$, and the second had a mean decay time constant of $25.3 \pm 3.3 \mathrm{msec}$. The reversal potentials ranged from approximately $-65 \mathrm{mV}$ to $-50 \mathrm{mV}$ for slow PSCs and $-10 \mathrm{mV}$ to $+15 \mathrm{mV}$ for rapid PSCs. The GABA receptor blocker bicuculline completely blocked the slow currents, whereas the AMPA receptor antagonist 6-cyano-7-nitroquinoxaline-2,3-dione (CNQX) completely blocked the fast currents. These findings indicate that the fast responses are EPSCs and the slow ones are IPSCs, so we used PSC decay times as a simple means to distinguish between glutamatergic and GABAergic synapses. Miniature EPSCs (mEPSCs) were measured in the absence of stimulation over a subsequent $60 \mathrm{sec}$ period. mEPSCs were detected semi-automatically and measured using the Mini Analysis Program (Synaptosoft). Synaptic depression was analyzed in neurons that had EPSC amplitudes $>0.5 \mathrm{nA}$, the median amplitude in synapsin III knock-out neurons. In these cells, trains of action potentials at 10 or $20 \mathrm{~Hz}$ were initiated after collection of spontaneous activity so as to minimize the effects of activity on spontaneous release frequency.

Optical measurements and analysis. Coverslips were mounted in a rapid-switching, laminar-flow perfusion and stimulation chamber on the stage of a laser-scanning confocal microscope. The total volume of the chamber was $\sim 75 \mu \mathrm{l}$ and was perfused at a rate of $1-1.5 \mathrm{ml} / \mathrm{min}$. Action potentials were evoked by passing $1 \mathrm{msec}$ current pulses yielding fields of $\sim 10 \mathrm{~V} / \mathrm{cm}$ through the chamber via platinum-iridium electrodes. Except as noted otherwise, cells were superfused continuously at room temperature in a saline solution consisting of (in mM): $119 \mathrm{NaCl}, 2.5 \mathrm{KCl}, 2$ $\mathrm{CaCl}_{2}, 2 \mathrm{MgCl}_{2}, 25 \mathrm{HEPES}, \mathrm{pH} 7.4$, and 30 glucose plus $10 \mu \mathrm{M} \mathrm{CNQX}$ 
(Research Biochemicals, Natick, MA) and $50 \mu \mathrm{M}$ D,L-2-amino-5phosphonovaleric acid (AP-5; Research Biochemicals). FM1-43 (Molecular Probes, Eugene, OR) was used at a concentration of $15 \mu \mathrm{M}$. Laserscanning fluorescence and differential interference contrast images were acquired simultaneously at a spatial sampling of $125 \mathrm{~nm}$ per pixel and a dwell time of $2 \mathrm{msec}$ per pixel through a $40 \times 1.3$ numerical aperture Zeiss Fluar objective (Oberkochen, Germany), using a custom-built laser-scanning microscope. Quantitative measurements of fluorescence intensity at individual synapses were obtained by averaging a $4 \times 4$ area of pixel intensities centered on the optical center of a given fluorescent punctum. Individual puncta were selected by hand, and the optical center of mass used to center the measurement box was computed over a slightly larger area (typically $8 \times 8$ pixels). Large puncta, typically representative of clusters of smaller synapses, were rejected during the selection procedure as were any puncta that were not clearly discernible in all test episodes. At least 50 boutons from each genotype were imaged in each of these experiments.

Immunocytochemical procedures. Cultures were fixed for $20 \mathrm{~min}$ with $4 \%$ paraformaldehyde in PBS containing $0.12 \mathrm{~m}$ sucrose. They were then permeabilized in $0.3 \%$ Triton X-100 in PBS for $4 \mathrm{~min}$ and rinsed twice in PBS. The cells were preincubated in $10 \%$ BSA in PBS for $1 \mathrm{hr}$ at $37^{\circ} \mathrm{C}$ and exposed to the primary antibodies (diluted in 1\% BSA in PBS) overnight at $4^{\circ} \mathrm{C}$. Finally, the cultures were rinsed in PBS and incubated with secondary antibodies for $1 \mathrm{hr}$ at $37^{\circ} \mathrm{C}$. Anti- $\alpha$-tubulin (clone DM1A; Sigma), rather than anti-tau, was used to stain the neurons, because tau is not compartmentalized to axons at this early stage of culture (Dotti et al., 1987; Ferreira et al., 1989). Pictures were taken using TMAX 400 ASA film (Kodak, Rochester, NY) on a Nikon microscope equipped with a photographic camera. Films were scanned using a Polaroid Sprint SCAN 35 scanner.

\section{RESULTS}

\section{Generation of synapsin III knock-out mice}

Previous reports have shown that deleting exon 1 of synapsin I or synapsin II resulted in null mutations (Rosahl et al., 1993, 1995; Li et al., 1995; Takei et al., 1995). We cloned the genomic DNA containing exon 1 of the murine synapsin III gene and found that the exon-intron boundary for synapsin III was identical to those for synapsins I and II. This led us to use the same gene targeting strategy to generate the synapsin III knock-out mice. A targeting vector was constructed to replace exon 1 of synapsin III with a neomycin-resistance gene in reverse orientation (Fig. 1A). After homologous recombination in ES cells, the targeted allele should contain a $6.8 \mathrm{~kb}$ EcoRI fragment, whereas the wild-type allele should have an $8.1 \mathrm{~kb}$ EcoRI fragment (Fig. 1A). From the 10 positive ES cell clones that were obtained, 4 were injected into C57BL/6 blastocysts and produced germ-line transmission. Tail DNA from F1 littermates of intercrosses between heterozygotes were digested with EcoRI and blotted with an external probe or a probe for the neomycin resistance gene. Disruption of the synapsin III gene in the homozygote $(-/-)$ was demonstrated by the presence of a single band of the correct size $(6.8 \mathrm{~kb})$, which contained the neomycin resistance gene (Fig. $1 B$ ). The absence of synapsin III protein in the knock-outs was revealed by Western blots of total brain homogenates. Two independent antibodies, RU486 (Fig. 1C) and RU482 (data not shown), which were raised against different regions in the $\mathrm{C}$ terminus of synapsin III, were used to measure synapsin III protein levels. In heterozygous mice $(+/-)$, the expression level of synapsin III was greatly reduced in comparison with wild-type mice, reminiscent of the situation in synapsin $\mathrm{I}^{+/-}$or synapsin $\mathrm{II}^{+/-}$mice (Rosahl et al., 1993, 1995; Li et al., 1995). No changes were observed in the level of expression of other synaptic vesicle proteins, namely, synapsin I, synapsin II, syntaxin, synaptotagmin, or synaptophysin (Fig. 1C).

A Mendelian distribution of synapsin III knock-out mice among offspring from heterozygote matings showed that no lethality resulted from disruption of the synapsin III gene. There
A
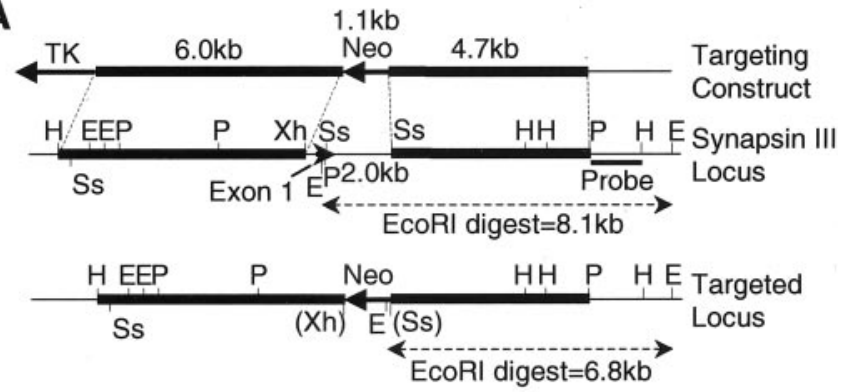

B

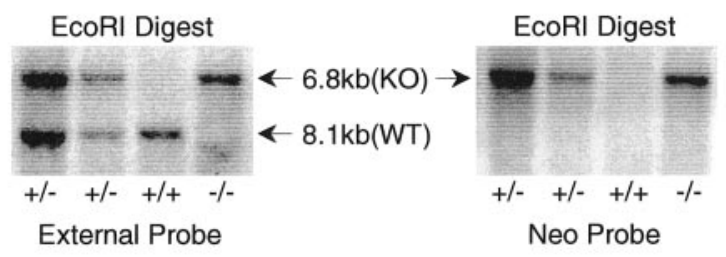

C

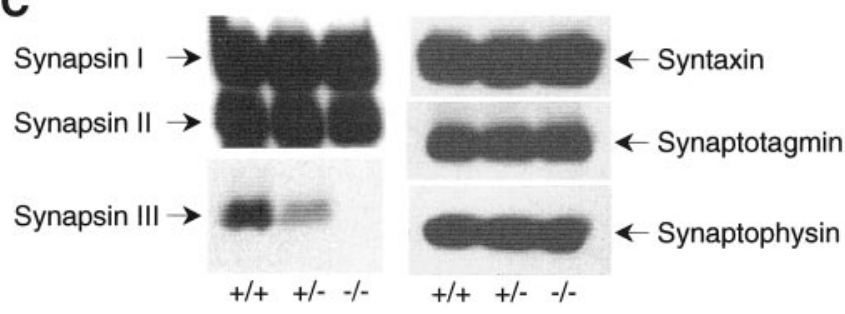

Figure 1. Generation of synapsin III knock-out mice. $A$, Synapsin III targeting vector consisted of a 6.0 and a $4.7 \mathrm{~kb}$ homologous region flanking the neo ${ }^{\mathrm{R}}$ gene to replace the $2.0 \mathrm{~kb}$ fragment containing exon 1 (top line). Restriction map, location of exon 1, and the external probe are shown in the middle line. Structure of the synapsin III locus after homologous recombination is shown in the bottom line. Restriction enzyme sites are as indicated: H, HindIII; E, EcoRI; P, PstI; Xh, XhoI; Ss, SstI. Sizes of the $E c o$ RI fragments in the wild-type and targeted alleles are shown. $B$, Southern blot analysis of EcoRI-digested tail DNA from wild-type $(+/+)$, heterozygous $(+/-)$, and homozygous $(-/-)$ littermates using the external probe (left). The same blot was stripped and reprobed with the neo probe (right). $C$, Western blot analysis of expression levels of synapsin III and a few other synaptic vesicle proteins in brain homogenate from wild-type $(+/+)$, heterozygous $(+/-)$, and homozygous $(-/-)$ littermates. $K O$, Knock-out; $W T$, wild type.

were also no gross changes in the anatomy or behavior of synapsin III knock-out mice. In contrast to synapsin I and synapsin II knock-out mice, seizures were not observed in synapsin III knock-out mice of any age. Furthermore, susceptibility to kainate- or pentylenetetrazole-induced seizures was not different between the wild-type and synapsin III knock-out mice (data not shown). All four independently derived lines of knock-out mice exhibited virtually identical phenotypes.

\section{Altered synaptic vesicle recycling in synapsin III-deficient mice}

Given the roles of synapsins I and II in regulation of neurotransmitter release, we examined synaptic vesicle trafficking in presynaptic terminals of synapsin III knock-out mice. Cultured hippocampal neurons were prepared from synapsin III knock-out mice or wild-type controls. Seven paired experiments were performed with cultures from wild-type and synapsin III knock-out littermates. A train of 600 action potentials (APs) was delivered $(10 \mathrm{~Hz})$ to these neurons in the presence of the amphipathic fluorescent probe FM1-43 (Betz et al., 1992) to label recycling 

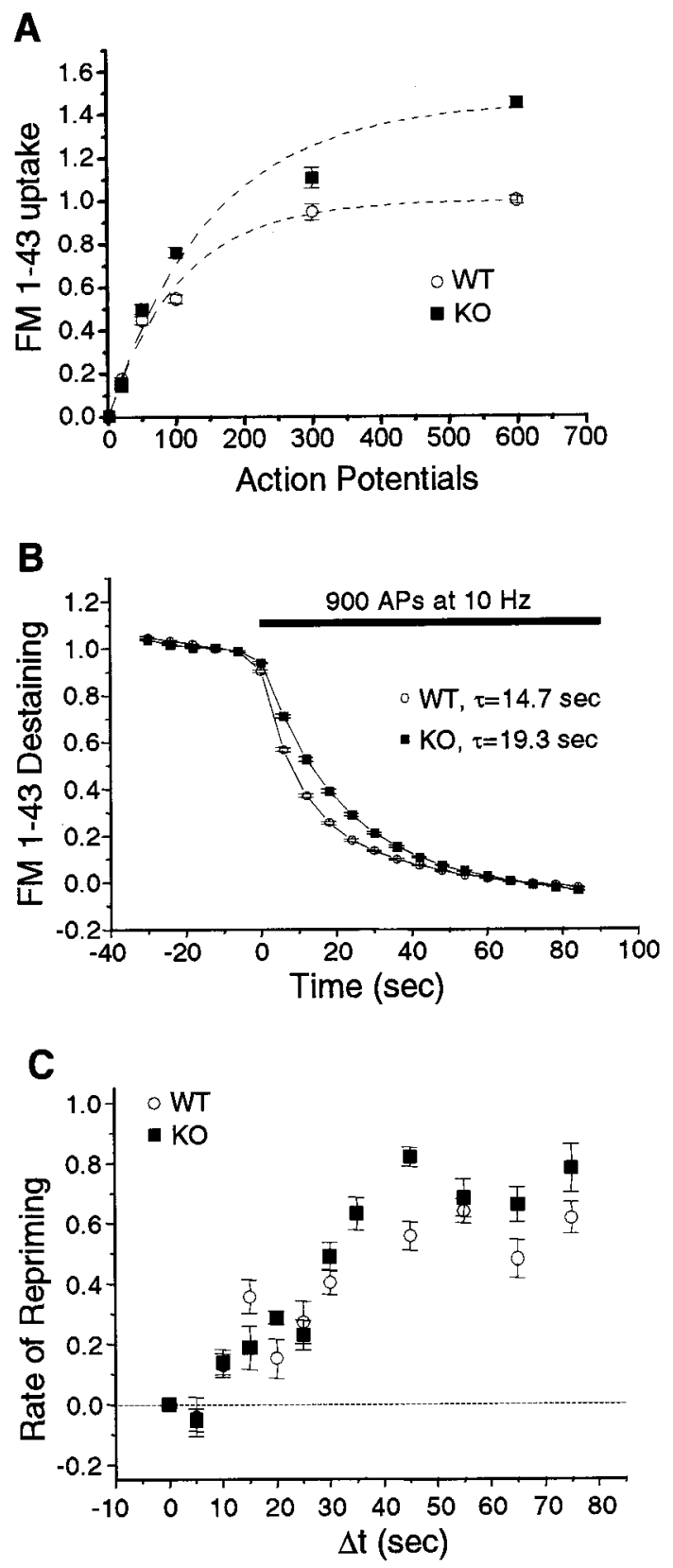

Figure 2. Synaptic vesicle recycling profiles in wild-type and synapsin III-deficient terminals. $A$, Size of the recycling pool of synaptic vesicles as measured by FM1-43 uptake in response to action potential stimuli. $B$, Release kinetics as measured by the time course for the depletion of FM1-43 staining. Time constants $(\tau)$ for wild-type and synapsin IIIdeficient boutons are shown. $C$, Vesicle repriming rates for wild-type and synapsin III-deficient terminals.

vesicles with the dye through endocytosis (Ryan et al., 1996). Another train of 900 APs $(10 \mathrm{~Hz})$ was then applied to the neuron to evoke the exocytotic release of neurotransmitters and concurrent loss of FM1-43. The maximal magnitude of the loss of dye fluorescence $(\Delta F)$, which reflects the size of the recycling vesicle pool (Ryan et al., 1996), was increased by $42.3 \pm 2.7 \%$ in synapsin III knock-out mice compared with that of wild-type littermate controls (Fig. 2A). Thus, loss of synapsin III increases the size of the recycling pool of synaptic vesicles.

To analyze vesicle pool release dynamics, synaptic vesicles were first loaded by stimulation with 600 APs $(20 \mathrm{~Hz})$ in the presence of FM1-43, and vesicle fusion was then measured in the absence of dye by stimulating the neurons with an additional 900 APs (10 $\mathrm{Hz}$ ). The average time course for the loss of fluorescence at each bouton was well described by an exponential function. The rate of dye release was slower in synapsin III knock-out neurons than in controls (Fig. $2 B$ ). The time constant, $\tau$, for the exponential loss of FM1-43 was $14.7 \mathrm{sec}$ in wild-type neurons but $19.3 \mathrm{sec}$ for synapsin III-deficient boutons.

The slower kinetics of dye loss might reflect a decrease in the probability that an AP can trigger vesicle fusion in the synapsin III knock-out neurons. However, given that the recycling vesicle pool is larger in the knock-out neurons, the increase in $\tau$ may simply reflect the presence of more vesicles to release over the course of the stimulus. To examine this possibility, the average number of vesicles fused per action potential was estimated from $\tau$ and the initial pool size. Assuming that $N$ vesicles reside in the recycling pool of wild-type neurons, then synapsin III knock-out neurons have $1.42 \mathrm{~N}$ vesicles. The measurements shown in Figure $2 B$ indicate that on average, 193 APs were required to release $1.42 N \times(1-1 / e)$ vesicles in synapsin III knock-out synapses and 147 APs in the wild-type synapses to release $N \times(1-1 / e)$ vesicles. These values suggest that each AP in the stimulus train released on average $0.0046 N[1.42 N \times(1-1 / e) / 193]$ vesicles in synapsin III $^{-/-}$terminals and $0.0043 N[N \times(1-1 / e) / 147]$ vesicles in the wild-type terminals. Thus, roughly the same number of vesicles fused per AP in wild-type and knock-out mice. These results suggest that knock-out of synapsin III did not greatly alter the probability that a synaptic vesicle will fuse after an AP.

It is also possible that synapsin III is involved in the repriming steps that precede vesicle fusion. To measure vesicle repriming in synapsin III knock-out neurons, we used a protocol described previously (Ryan and Smith, 1995; Ryan et al., 1996). FM1-43 was loaded into terminals via a train of APs $(20 \mathrm{~Hz})$, and this stimulus outlasted the $5 \mathrm{sec}$ period of FM1-43 treatment by a variable length of time, $\Delta t$. The change in fluorescence intensity at $\Delta t=0$ was designated $\Delta F_{0}$. At later times, dye fluorescence $\left(\Delta F_{\Delta t}\right)$ was lost by the subsequent fusion of dye-filled vesicles. The time required for these dye-filled vesicles to become fusion competent, or reprimed, was determined for each $\Delta t$ by calculating the fraction of fluorescence depleted by the firing of APs, namely the ratio $\left(\Delta F_{0}-\Delta F_{\Delta t}\right) / \Delta F_{0}$. As shown in Figure $2 C$, very little repriming occurred during the first $10 \mathrm{sec}$ after removal of extracellular FM1-43. However, the extent of dye loss increased steadily over the next $60 \mathrm{sec}$ as labeled vesicles were reprimed and became available for fusion. The time course of repriming was very similar in wild-type and synapsin III knock-out neurons. Furthermore, in each case, the extent of repriming reached a plateau near $70 \%$. This suggests that in presynaptic terminals of both wild-type and knock-out neurons, $\sim 30 \%$ of endocytosed vesicles were unavailable for recycling within $60 \mathrm{sec}$. These results indicate that synapsin III is not involved in synaptic vesicle repriming.

In summary, FM1-43 measurements indicate that synapsin III limits the size of the recycling pool but has little effect on the probability of vesicle fusion or the rate at which synaptic vesicles are reprimed.

\section{Altered neurotransmitter release in synapsin III-deficient mice}

To determine whether these changes in vesicle trafficking in synapsin III knock-out mice lead to changes in neurotransmitter release, patch-clamp recordings were made from cultured hip- 
A
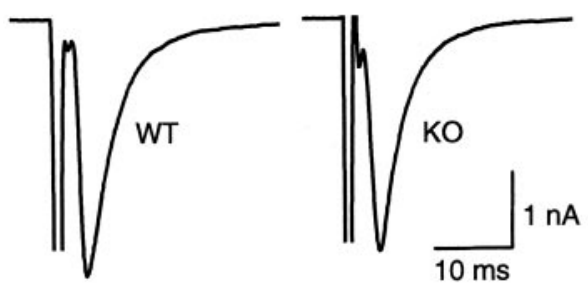

C
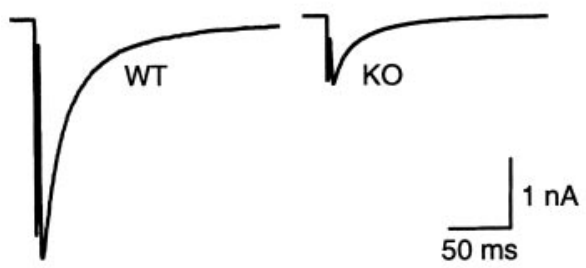

B

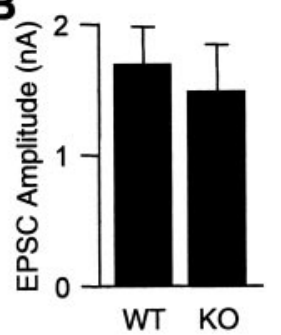

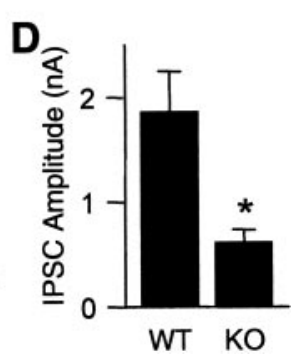

Figure 3. Evoked neurotransmitter release in wild-type and synapsin III-deficient neurons. $A$, Representative EPSC traces from wild-type $(W T)$ and synapsin III-deficient neurons $(K O)$. B, EPSC amplitude in neurons from wild-type $(W T)$ or synapsin III knock-out mice $(K O)(n=$ 71 and 69, respectively). $C$, Representative IPSC traces from wild-type and synapsin III-deficient neurons. $D$, IPSC amplitude in neurons from wild-type or synapsin III knock-out mice ( $n=15$ and 28, respectively).

pocampal neurons. In these experiments, microisland cultures were used to induce the formation of autaptic synaptic connections on individual neurons (Bekkers and Stevens, 1991). Synaptic transmission could then be evoked by briefly depolarizing a neuron while using the same patch pipette to measure the resulting EPSCs. In this way, we recorded EPSCs from 71 wild-type neurons and 69 mutant neurons in four cultures prepared from littermates. The amplitude of evoked EPSCs was not significantly different between synapsin III-deficient neurons and wild-type neurons (Fig. 3A,B). The decay time constant of EPSCs and the total charge associated with these currents were also the same in synapsin III-deficient and wild-type control neurons. We examined how EPSC amplitude varied with development and found no difference between wild-type and knock-out neurons between days 7 and 9 in culture. Thus, the strength and kinetics of excitatory synaptic transmission are preserved in the absence of synapsin III. Some of the neurons in cultures were interneurons, and we used the same procedures to record IPSCs from 15 wild-type neurons and 26 mutant neurons. In contrast to what was observed at excitatory synapses, there was a significant reduction in the amplitude of IPSCs in synapsin III-deficient neurons compared with IPSCs of wild-type neurons (Fig. 3C,D). This decrease was evident in neurons of various ages (data not shown). These results indicate that synapsin III plays a more prominent role in inhibitory synaptic transmission than in excitatory synaptic transmission.

To measure the characteristics of individual quanta, we analyzed mEPSCs resulting from spontaneous release. The amplitude of mEPSCs in synapsin III $^{-1-}$ neurons was slightly but significantly larger than in wild-type neurons $(p<0.05)$ (Fig. $4 A, B)$. The frequency of mEPSCs was slightly higher in mutant neurons, although not significantly different from the wild-type controls (Fig. 4A,C). The quantal content of evoked transmission was calculated by dividing the amplitude of evoked responses by that of the single quantal response (mEPSCs). For those neurons

A
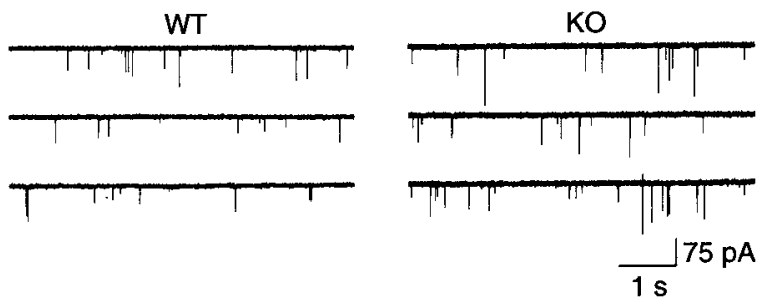

B

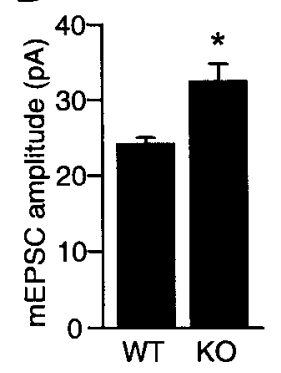

C
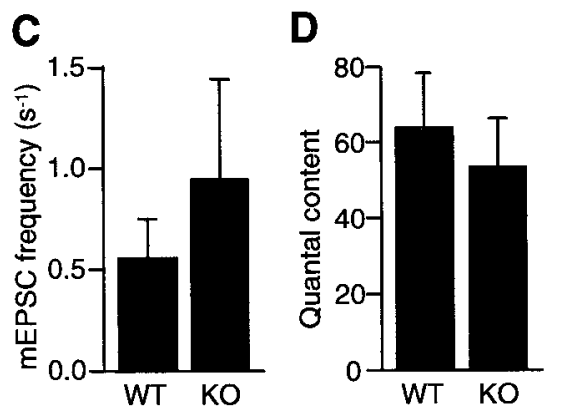

Figure 4. Spontaneous neurotransmitter release in wild-type and synapsin III-deficient neurons. $A$, Representative mEPSC traces from wild-type $(W T)$ and synapsin III-deficient neurons $(K O) . B$, mEPSC amplitude in neurons from wild-type or synapsin III knock-out mice. $C$, mEPSC frequency in neurons from wild-type or synapsin III knock-out mice. $D$, Quantal content of EPSCs in neurons from wild-type $(n=29)$ or synapsin III knock-out $(n=32)$ mice.
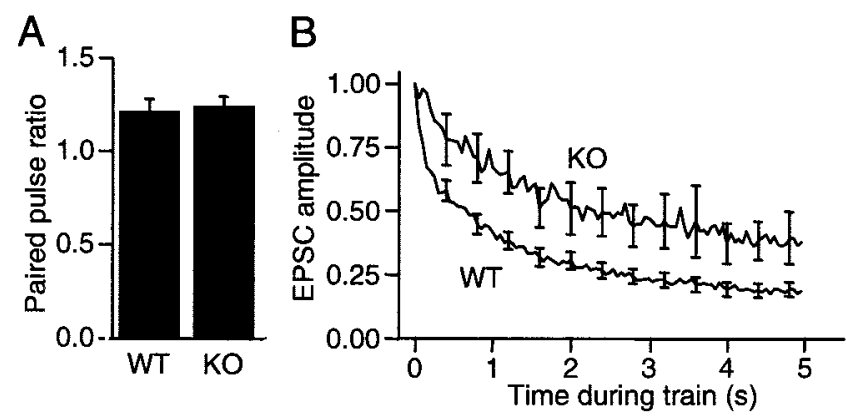

Figure 5. Paired-pulse facilitation and synaptic depression at wild-type and synapsin III-deficient synapses. $A$, Paired-pulse facilitation was measured with the second pulse applied $20 \mathrm{msec}$ after the first one. $B$, Synaptic depression was evoked by stimulating the neurons with a $20 \mathrm{~Hz}$ train. EPSCs were normalized to the initial response to measure the extent of synaptic depression.

in which both EPSCs and mEPSCs were measured, the quantal content was not significantly different between wild-type and synapsin III-deficient neurons (Fig. 4D). Thus, despite the fact that the absence of synapsin III increases the number of recycling synaptic vesicles and increases the response to release of individual vesicles, glutamatergic synaptic transmission remains grossly unaltered in synapsin III knock-out neurons stimulated at low frequencies.

To study whether short-term synaptic plasticity was affected by the deletion of synapsin III, we measured synaptic facilitation and depression. Facilitation was examined by firing pairs of APs separated by an interval of $20 \mathrm{msec}$. As shown in Figure $5 A$, there was no significant difference in synaptic facilitation between wildtype and synapsin III-deficient neurons. This is consistent with other observations that synapsins are involved in facilitation to only a limited extent (Rosahl et al., 1995) or not at all (Hilfiker et al., 1998). When neurons were stimulated with prolonged trains of 
APs $(20 \mathrm{~Hz})$, synaptic depression caused the amplitudes of evoked EPSCs to gradually decline as the supply of releasable vesicles was depleted. After $5 \mathrm{sec}$ of stimulation, transmission at wild-type synapses was depressed by $81 \pm 2 \%$, whereas depression was significantly less, only $62 \pm 10 \%$, in synapsin $\mathrm{III}^{-/-}$ neurons $(p<0.05)$. Given that EPSCs evoked by low-frequency stimulation were essentially unaffected by deletion of synapsin III (Fig. 3), this decrease in depression indicates that synapsin III normally makes excitatory synapses more sensitive to periods of high activity. The rate of refilling of the readily releasable pool is $\sim 3 \mathrm{sec}$ at active hippocampal terminals (Stevens and Wesseling, 1999), whereas the time constants for depression range from $0.8 \pm 0.06 \mathrm{sec}$ for wild-type neurons to $1.52 \pm 0.08 \mathrm{sec}$ in the synapsin III knock-out neurons. The similarity of the time constants for depression and refilling suggests that the increased recycling vesicle pool observed in FM1-43 studies could contribute to the slowing of depression in the knock-out neurons. A larger pool of vesicles would be depleted more slowly during a train of APs if vesicles fused with approximately the same probability, as was indicated by the lack of change in quantal content of EPSCs evoked at low frequency.

\section{Ultrastructure of synapsin III-deficient hippocampal synapses}

We used electron microscopy to study the structure of synapsin III-deficient synapses. Because synapsin III is very highly expressed in MFTs (H. T. Kao, J. Feng, P. Greengard, and V. A. Pieribone, unpublished observations), we examined the stratum lucidum of the CA3 region of hippocampi from wild-type and synapsin III knock-out littermates. As shown in Figure 6 and Table 1, there was no significant difference between experimental groups in the net area occupied by MFT profiles or in the area occupied by mitochondria and spine profiles. Compared with wild-type mice, there was a significant, although small, increase in the number of spine profiles embedded within MFTs of synapsin III knock-outs. The density of synaptic vesicles within MFTs of wild-type neurons was very similar to that measured in synapsin III knock-outs.

Because mossy fiber synapses contain multiple giant spines and multiple active zones, it is difficult to accurately quantify the spatial distribution of synaptic vesicles within these terminals. We therefore measured the distribution of synaptic vesicles in CA1 presynaptic terminals in the stratum radiatum layer of the hippocampus. As shown in Figure 7 and Table 2, there was no significant difference in the spatial distribution of vesicles within these terminals. Thus, although our functional measurements indicate that loss of synapsin III affects the number of vesicles in the recycling pool, this difference is apparently not reflected in the number or spatial distribution of synaptic vesicles within presynaptic terminals.

\section{Role of synapsin III in axon outgrowth}

Previous studies showed that depletion of synapsin III, by treating cultured hippocampal neurons with antisense RNA, impaired axon extension and enlarged growth cones (Ferreira et al., 2000). To further consider the role of synapsin III in these processes, we examined neurite outgrowth in hippocampal neurons cultured from wild-type and synapsin III knock-out mice. These neurons can be classified developmentally on the basis of their morphology (Goslin and Banker, 1991). Neurons at stage 1 have only lamellipodia extending from the cell bodies, without any processes. At stage 2, minor processes extend out from the cell bodies. Within

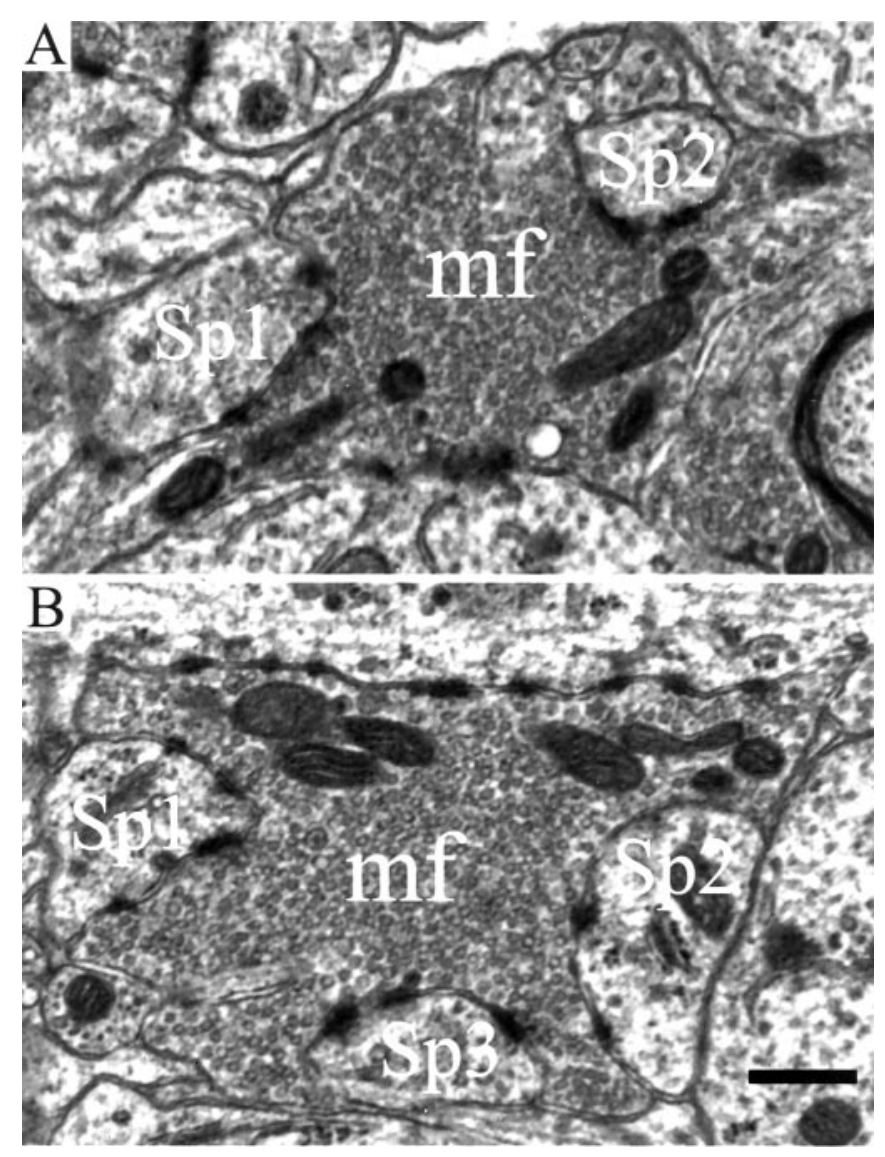

Figure 6. Effect of synapsin III deletion on the ultrastructure of MFTs in the stratum lucidum of the dorsal hippocampal CA3 region. $A$, Representative MFT ( $m f$ ) of a wild-type mouse perforated with two giant spines $(S p)$ of CA3 proximal apical dendrites. The terminal was densely packed with synaptic vesicles and some electron-dense mitochondrial profiles. $B$, Representative MFTs of a synapsin III knockout mouse perforated with three giant spines $(S p)$. $m f$, Mossy fiber terminal; $S p$, spine. Scale bar, $1 \mu \mathrm{m}$.

Table 1. Quantification of ultrastructural variables in mossy fiber terminals from wild-type and synapsin III knock-out mice

\begin{tabular}{lcc} 
Morphological variables & Wild type & Knock-out \\
\hline Vesicle density, number/ $\mu \mathrm{m}^{3}$ & $5608 \pm 137$ & $5878 \pm 106$ \\
MFT profile area, $\mu \mathrm{m}^{2}$ & $3.89 \pm 0.14$ & $4.03 \pm 0.17$ \\
Mitochondrial profile area, $\mu \mathrm{m}^{2}$ & $0.24 \pm 0.02$ & $0.26 \pm 0.03$ \\
Spine profile area, $\mu \mathrm{m}^{2}$ & $0.82 \pm 0.05$ & $0.92 \pm 0.05$ \\
Net MFT area, $\mu \mathrm{m}^{2}$ & $2.85 \pm 0.11$ & $2.86 \pm 0.12$ \\
Number of mitochondria & & \\
$\quad$ profiles/MFT profile & $4.18 \pm 0.45$ & $4.30 \pm 0.44$ \\
Number of spines/MFT profile & $3.36 \pm 0.11$ & $4.13 \pm 0.27^{*}$ \\
\hline
\end{tabular}

Data are expressed as mean \pm SEM. ${ }^{*} p<0.05$, compared with wild type by two-tailed unpaired Student's $t$ test.

$24 \mathrm{hr}$, one of these processes has elongated further and become an axon (stage 3). Dendritic outgrowth occurs afterward for a few days (stage 4 ), and by $\sim 7 \mathrm{~d}$ after plating, neurons achieve a mature morphology and have synaptic contacts (stage 5).

To examine neurite outgrowth, embryonic day 16 hippocampal neurons were dissociated from wild-type or synapsin III knockout mice, and $24 \mathrm{hr}$ later anti- $\alpha$-tubulin antibody was used to visualize their neurites. The identification of a given process as an 

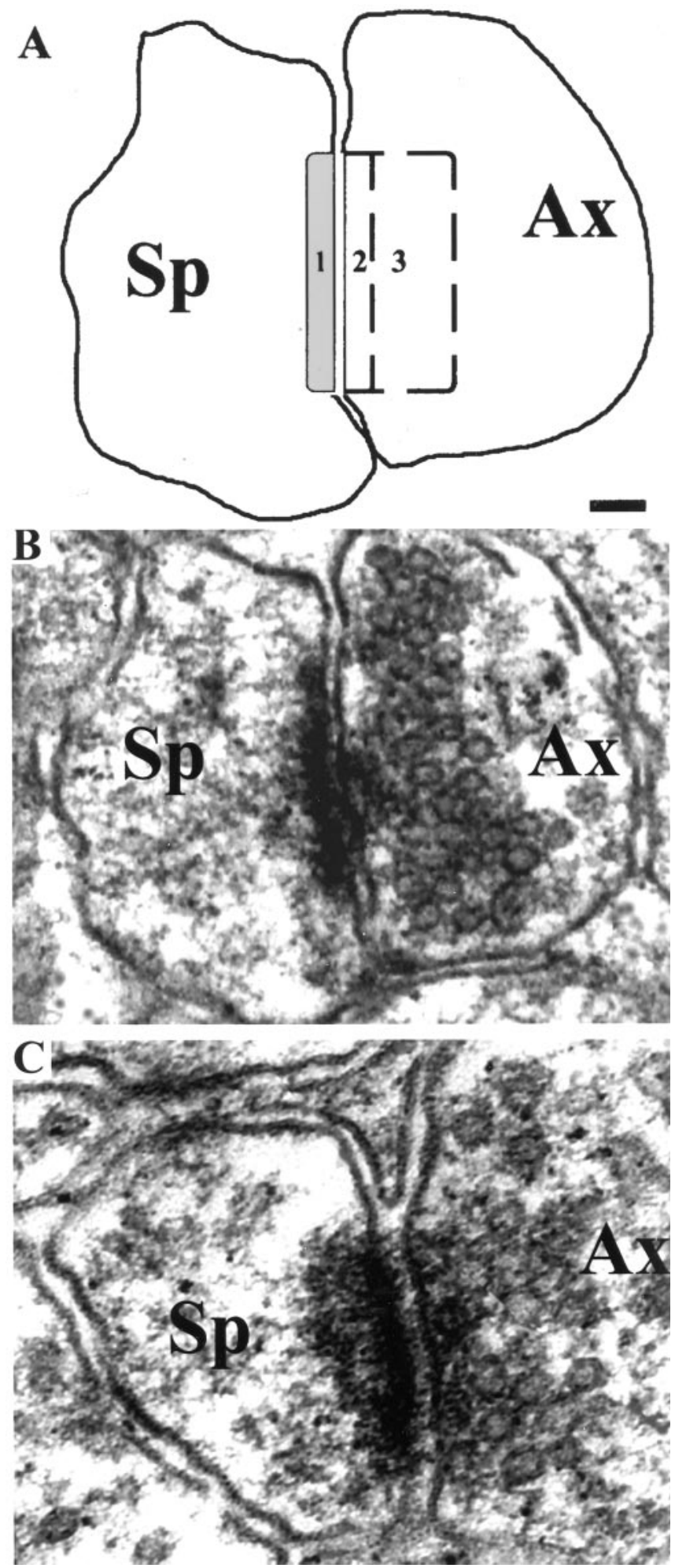

Figure 7. Effect of synapsin III deletion on the ultrastructure of presynaptic terminals within the stratum radiatum of the hippocampal CA1 region. $A$, Tracing of a representative axon terminal and adjacent postsynaptic spine forming an excitatory synapse. The drawing illustrates the postsynaptic density (1) and the areas where the number of vesicles was estimated: within $50 \mu \mathrm{m}$ of the presynaptic membrane associated with the active zone (2) and between 50 and $200 \mu \mathrm{m}$ of the presynaptic membrane (3). Docked vesicles were those physically attached to the presynaptic membrane. $B$, Representative excitatory synapse of a wild-type mouse. $C$, Representative excitatory synapse of a synapsin III knock-out mouse. $A x$, Axon terminal; $S p$, spine. Scale bar, $0.1 \mu \mathrm{m}$.
Table 2. Distribution of synaptic vesicles in CA1 terminals from wildtype and synapsin III knock-out mice

\begin{tabular}{llll} 
& Docked at $\mathrm{AZ}$ & $0-50 \mathrm{~nm}$ from $\mathrm{AZ}$ & $50-200 \mathrm{~nm}$ from $\mathrm{AZ}$ \\
\hline Wild type & $1.38 \pm 0.19$ & $8.19 \pm 0.39$ & $18.86 \pm 0.57$ \\
Knock-out & $1.26 \pm 0.23$ & $7.81 \pm 0.14$ & $18.72 \pm 0.16$ \\
\hline
\end{tabular}

Data are expressed as mean \pm SEM. AZ, Active zone.
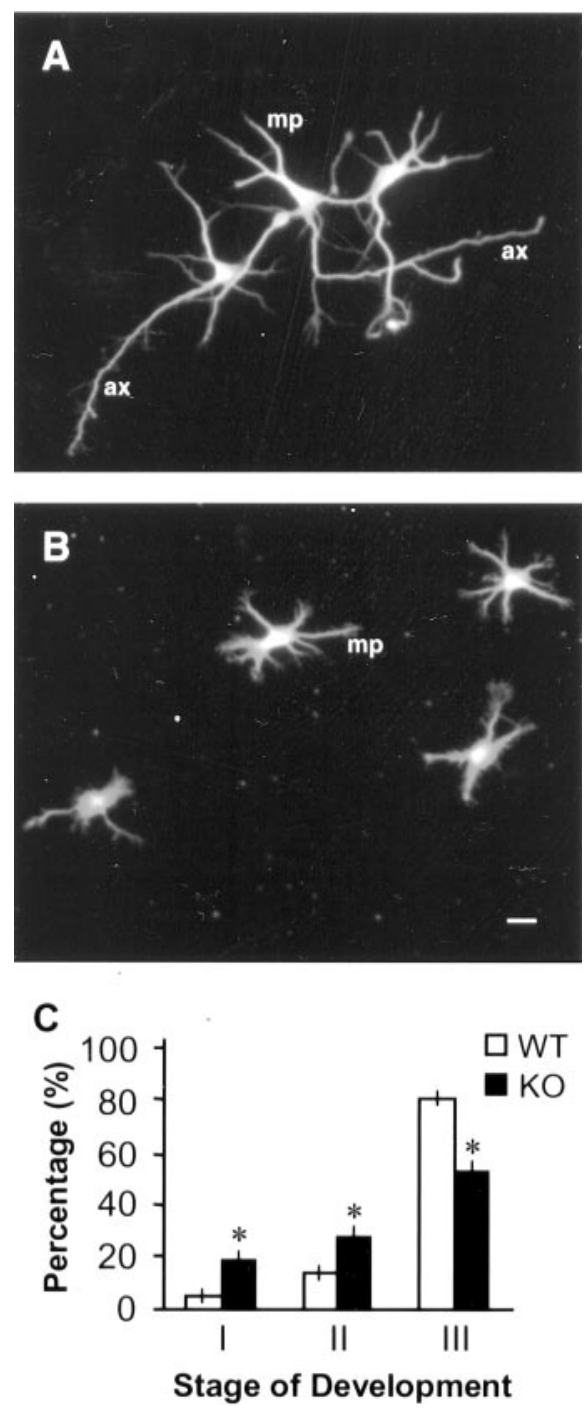

Figure 8. Delayed axon outgrowth in synapsin III-deficient neurons. $A$, Wild-type neurons were cultured for $24 \mathrm{hr}$ and stained with anti- $\alpha$ tubulin. $B$, Synapsin $\mathrm{III}^{-/-}$neurons were cultured for $24 \mathrm{hr}$ and stained with anti- $\alpha$-tubulin. $m p$, Minor process; $a x$, axon. $C$, Distribution of wild-type and synapsin III $^{-/-}$neurons at different stages of development.

axon at these early stages of development is made by morphological criteria as described previously (Dotti and Banker, 1987; Dotti et al., 1988). In addition, it has been shown that minor processes only start to elongate and differentiate as dendrites $4 \mathrm{~d}$ after plating (Dotti et al., 1988). As shown in Figure 8, axon outgrowth was much delayed in synapsin III-deficient neurons. Most wild-type neurons were at stage 3 , and only a small fraction remained at stage 1 or 2 . In contrast, a smaller fraction of synapsin III $^{-/-}$neurons were at stage 3 , and large numbers of neurons were at earlier stages of development (Fig. 8C). Synapsin 
III-deficient neurons had delayed axon extension, as well as enlarged growth cones and many lamellipodia, a pattern that is very similar to the appearance of wild-type neurons treated with synapsin III antisense RNA (Ferreira et al., 2000). However, when synapsin III-deficient neurons were cultured for $2 \mathrm{~d}$, there were no significant differences in their developmental profile in comparison with wild-type neurons (data not shown). These results indicate that synapsin III plays a very specific role in the early development of axons, being required during the first $24 \mathrm{hr}$ of axon extension. The physiological impact of this effect on early axon development in vivo awaits further analyses.

\section{DISCUSSION}

In this paper, we provide evidence indicating unique roles for synapsin III in the regulation of neurotransmitter release and in the initial outgrowth of axons. Through the generation and analysis of synapsin III knock-out mice, we found that synapsin III, the most recently identified member of the synapsin family, functions in vivo to limit the size of the recycling pool of synaptic vesicles and to accentuate synaptic depression during periods of rapid activity. At excitatory synapses, loss of synapsin III did not affect the average number of vesicles fused per action potential or synaptic transmission evoked by low frequencies of repetitive stimulation. However, transmission was substantially weaker at inhibitory GABAergic synapses in the absence of synapsin III. In addition to these roles in regulation of neurotransmitter release, synapsin III also appears to play a distinct and nonredundant function in the initial stage of axon outgrowth.

One of the most intriguing functions of synapsin III is its ability to limit the number of vesicles that undergo exocytotic-endocytotic recycling during sustained synaptic activity. By measuring the loss of FM1-43 fluorescence, we can operationally define the size of the recycling pool of synaptic vesicles. The increase of this pool in synapsin III-deficient terminals suggests that synapsin III plays a key role in keeping vesicles out of the recycling pool. Synapsin III, like other synapsins, interacts with phospholipids on synaptic vesicles (Hosaka et al., 1999). In this manner, synapsin III, along with synapsins I and II, may serve to tether vesicles to the cytoskeleton and keep them from recycling during synaptic activity (Benfenati et al., 1989, 1993; Hosaka et al., 1999). Removal of synapsin III by gene targeting therefore may have allowed more vesicles to be recruited to participate in synaptic transmission.

The significant increase in the number of recycling vesicles in synapsin III-deficient neurons is in sharp contrast to what was seen in synapsin I knock-out mice (Ryan et al., 1996) or synapsin II knock-out mice (P. Chi, P. Greengard, and T. A. Ryan, unpublished observation), where the size of the recycling pool was significantly reduced. This dramatic difference may be explained by the apparent roles of synapsin I and II in the formation and stability of synaptic vesicles. Both synapsin I and synapsin II are normally expressed at very high levels on vesicles, but in the absence of either protein, the number of vesicles is reduced significantly (Li et al., 1995; Rosahl et al., 1995; Takei et al., 1995), and this reduction is most pronounced in synapsin I/II double knock-out mice (Rosahl et al., 1995). In contrast, the number of vesicles in synapsin III knock-out mice was quite similar to that in wild-type mice. Together, these results suggest that unlike synapsins I and II, the primary function of synapsin III is to anchor synaptic vesicles so that their recycling can be regulated, presumably through phosphorylation (Hosaka et al., 1999). Because the hippocampal culture that we used contained a mixed population of GABAergic $(\sim 25 \%)$ and glutamatergic $(\sim 75 \%)$ neurons (P. Chi and T. A. Ryan, unpublished observations), our results on vesicle trafficking were the average of effects of inhibitory and excitatory terminals. Future studies, using autaptic cultures for example, would allow us to examine the potentially different effects in vesicle recycling between GABAergic and glutamatergic boutons in synapsin III knock-out mice.

A larger recycling vesicle pool in synapsin III-deficient terminals might have been expected to increase the strength of synaptic transmission. However, we found that the quantal content of glutamatergic EPSCs was unchanged during low-frequency synaptic activation. This implies that either the number and release probability of the recycling vesicles ready for fusion remain unchanged or, if more recycling vesicles are available for immediate release, the probability of release per vesicle decreases. In fact, we found that the frequency of spontaneous release from synapsin III-deficient terminals did not change and that the number of vesicles fused per action potential, calculated both from FM1-43 destaining rates and from EPSC quantal content, did not change. Thus, knock-out of synapsin III does not alter basal transmission at excitatory synapses, and its primary functional phenotype is altered synaptic plasticity during periods of high synaptic activity, which is similar to what has been reported after knock-out of synapsins I or II (Li et al., 1995; Rosahl et al., 1995; Takei et al., 1995).

In contrast, the amplitudes of IPSCs at GABAergic synapses were strongly reduced in synapsin III knock-out mice. This suggests that the function of synapsin III in inhibitory terminals may be different from that at excitatory synapses. Alternatively, GABAergic neurotransmission, which typically involves much higher action potential frequencies than at glutamatergic synapses, may naturally rely on a different distribution of vesicles between reserve and recycling pools that is greatly sensitive to the loss of synapsin III. Inhibitory synaptic transmission is also impaired in mice lacking synapsin I, a defect that presumably contributes to increased seizure propensity in those mice (Terada et al., 1999). However, we did not observe any spontaneous seizures in synapsin III knock-out mice, despite the substantial impairment of inhibitory synaptic transmission. In addition, seizure susceptibility to kainate or the GABAergic antagonist pentylenetetrazole was similar in synapsin III knock-out mice and sex-matched wild-type littermates. Thus, there is not a tight coupling between the magnitude of hippocampal synaptic inhibition and seizure propensity.

Our studies revealed a specific requirement for synapsin III at an initial stage of axon outgrowth. Synapsin III has a unique developmental expression pattern, being expressed at its highest levels during the first few days in culture and diminishing precipitously afterward. In addition, synapsin III is highly localized in growth cones and appears at extrasynaptic sites, quite unlike the distinct, synaptic localization of synapsins I and II (Ferreira et al., 2000). The recovery of axon morphology in later stages of development indicates that other synapsins may eventually compensate for the absence of synapsin III. The short span of time during which synapsin III deficiency is reflected in altered morphology is a remarkable demonstration of the temporal regulation of gene expression and function. Together, these results strongly suggest that synapsin III plays a critical and nonredundant role in early axon development, well before the first synapses are established.

Through the generation and analyses of synapsin III knock-out mice, we have provided strong evidence for unique functions of synapsin III in both early axon development and the regulation of 
neurotransmitter release in mature synapses. These results should help to elucidate the complex and interdependent roles of synapsins in the nervous system.

\section{REFERENCES}

Bahler M, Greengard P (1987) Synapsin I bundles F-actin in a phosphorylation-dependent manner. Nature 326:704-707.

Bahler M, Benfenati F, Valtorta F, Greengard P (1990) The synapsins and the regulation of synaptic function. BioEssays 12:259-263.

Bekkers JM, Stevens CF (1991) Excitatory and inhibitory autaptic currents in isolated hippocampal neurons maintained in cell culture. Proc Natl Acad Sci USA 88:7834-7838.

Benfenati F, Valtorta F, Bahler M, Greengard P (1989) Synapsin I, a neuron-specific phosphoprotein interacting with small synaptic vesicles and F-actin. Cell Biol Int Rep 13:1007-1021.

Benfenati F, Valtorta F, Rubenstein JL, Gorelick FS, Greengard P, Czernik AJ (1992) Synaptic vesicle-associated $\mathrm{Ca}^{2+} /$ calmodulindependent protein kinase II is a binding protein for synapsin I. Nature 359:417-420.

Benfenati F, Valtorta F, Rossi MC, Onofri F, Sihra T, Greengard P (1993) Interactions of synapsin I with phospholipids: possible role in synaptic vesicle clustering and in the maintenance of bilayer structures. J Cell Biol 123:1845-1855.

Betz WJ, Mao F, Bewick GS (1992) Activity-dependent fluorescent staining and destaining of living vertebrate motor nerve terminals. J Neurosci 12:363-375.

Chi P, Greengard P, Ryan TA (2001) Synapsin dispersion and reclustering during synaptic activity. Nat Neurosci 4:1187-1193.

Dotti CG, Banker GA (1987) Experimentally induced alteration in the polarity of developing neurons. Nature 330:254-256.

Dotti CG, Banker GA, Binder LI (1987) The expression and distribution of the microtubule-associated proteins tau and microtubule-associated protein 2 in hippocampal neurons in the rat in situ and in cell culture. Neuroscience 23:121-130.

Dotti CG, Sullivan CA, Banker GA (1988) The establishment of polarity by hippocampal neurons in culture. J Neurosci 8:1454-1468.

Feng J, Yan Z, Ferreira A, Tomizawa K, Liauw JA, Zhuo M, Allen PB, Ouimet CC, Greengard P (2000) Spinophilin regulates the formation and function of dendritic spines. Proc Natl Acad Sci USA 97:9287-9292.

Ferreira A, Busciglio J, Caceres A (1989) Microtubule formation and neurite growth in cerebellar macroneurons which develop in vitro: evidence for the involvement of the microtubule-associated proteins, MAP-1a, HMW-MAP2 and Tau. Brain Res Dev Brain Res 49:215-228.

Ferreira A, Kao HT, Feng J, Rapoport M, Greengard P (2000) Synapsin III: developmental expression, subcellular localization, and role in axon formation. J Neurosci 20:3736-3744.

Goslin K, Banker G (1991) Rat hippocampal neurons in low-density culture. In: Culturing nerve cells (Banker G, Goslin K, eds), pp 251283. Cambridge, MA: MIT.

Greengard P, Valtorta F, Czernik AJ, Benfenati F (1993) Synaptic vesicle phosphoproteins and regulation of synaptic function. Science 259:780-785.

Hilfiker S, Schweizer FE, Kao HT, Czernik AJ, Greengard P, Augustine GJ (1998) Two sites of action for synapsin domain $\mathrm{E}$ in regulating neurotransmitter release. Nat Neurosci 1:29-35.

Hirokawa N, Sobue K, Kanda K, Harada A, Yorifuji H (1989) The cytoskeletal architecture of the presynaptic terminal and molecular structure of synapsin 1. J Cell Biol 108:111-126.

Hosaka M, Sudhof TC (1998) Synapsin III, a novel synapsin with an unusual regulation by $\mathrm{Ca} 2+$. J Biol Chem 273:13371-13374.

Hosaka M, Hammer RE, Sudhof TC (1999) A phospho-switch controls the dynamic association of synapsins with synaptic vesicles. Neuron 24:377-387.

Huttner WB, DeGennaro LJ, Greengard P (1981) Differential phosphorylation of multiple sites in purified protein I by cyclic AMPdependent and calcium-dependent protein kinases. J Biol Chem 256:1482-1488.

Jovanovic JN, Benfenati F, Siow YL, Sihra TS, Sanghera JS, Pelech SL, Greengard P, Czernik AJ (1996) Neurotrophins stimulate phosphorylation of synapsin I by MAP kinase and regulate synapsin I-actin interactions. Proc Natl Acad Sci USA 93:3679-3683.

Kao HT, Porton B, Czernik AJ, Feng J, Yiu G, Haring M, Benfenati F, Greengard P (1998) A third member of the synapsin gene family. Proc Natl Acad Sci USA 95:4667-4672.

Li L, Chin LS, Shupliakov O, Brodin L, Sihra TS, Hvalby O, Jensen V, Zheng D, McNamara JO, Greengard P (1995) Impairment of synaptic vesicle clustering and of synaptic transmission, and increased seizure propensity, in synapsin I-deficient mice. Proc Natl Acad Sci USA 92:9235-9239.

Llinas R, Gruner JA, Sugimori M, McGuinness TL, Greengard P (1991) Regulation by synapsin I and $\mathrm{Ca}^{2+}$-calmodulin-dependent protein kinase II of the transmitter release in squid giant synapse. J Physiol (Lond) 436:257-282.

Magarinos AM, Verdugo JM, McEwen BS (1997) Chronic stress alters synaptic terminal structure in hippocampus. Proc Natl Acad Sci USA 94:14002-14008.

Matsubara M, Kusubata M, Ishiguro K, Uchida T, Titani K, Taniguchi H (1996) Site-specific phosphorylation of synapsin I by mitogen-activated protein kinase and $\mathrm{Cdk} 5$ and its effects on physiological functions. J Biol Chem 271:21108-21113.

Pieribone VA, Shupliakov O, Brodin L, Hilfiker-Rothenfluh S, Czernik AJ, Greengard P (1995) Distinct pools of synaptic vesicles in neurotransmitter release. Nature 375:493-497.

Porton B, Kao HT, Greengard P (1999) Characterization of transcripts from the synapsin III gene locus. J Neurochem 73:2266-2271.

Rosahl TW, Geppert M, Spillane D, Herz J, Hammer RE, Malenka RC, Sudhof TC (1993) Short-term synaptic plasticity is altered in mice lacking synapsin I. Cell 75:661-670.

Rosahl TW, Spillane D, Missler M, Herz J, Selig DK, Wolff JR, Hammer RE, Malenka RC, Sudhof TC (1995) Essential functions of synapsins I and II in synaptic vesicle regulation. Nature 375:488-493.

Ryan TA, Smith SJ (1995) Vesicle pool mobilization during action potential firing at hippocampal synapses. Neuron 14:983-989.

Ryan TA, Li L, Chin LS, Greengard P, Smith SJ (1996) Synaptic vesicle recycling in synapsin I knock-out mice. J Cell Biol 134:1219-1227.

Stevens CF, Wesseling JF (1999) Identification of a novel process limiting the rate of synaptic vesicle cycling at hippocampal synapses. Neuron 24:1017-1028.

Sudhof TC, Czernik AJ, Kao HT, Takei K, Johnston PA, Horiuchi A, Kanazir SD, Wagner MA, Perin MS, De Camilli P, Greengard P (1989) Synapsins: mosaics of shared and individual domains in a family of synaptic vesicle phosphoproteins. Science 245:1474-1480.

Takei Y, Harada A, Takeda S, Kobayashi K, Terada S, Noda T, Takahashi T, Hirokawa N (1995) Synapsin I deficiency results in the structural change in the presynaptic terminals in the murine nervous system. J Cell Biol 131:1789-1800.

Terada S, Tsujimoto T, Takei Y, Takahashi T, Hirokawa N (1999) Impairment of inhibitory synaptic transmission in mice lacking synapsin I. J Cell Biol 145:1039-1048.

Tong G, Jahr CE (1994) Multivesicular release from excitatory synapses of cultured hippocampal neurons. Neuron 12:51-59.

Valtorta F, Greengard P, Fesce R, Chieregatti E, Benfenati F (1992) Effects of the neuronal phosphoprotein synapsin I on actin polymerization. I. Evidence for a phosphorylation-dependent nucleating effect. J Biol Chem 267:11281-11288. 